\title{
Antithrombin-III suppresses collagen-induced human platelets activation
}

Akiko Kojima ${ }^{1}$, Shigeru Akamatsu², Kumiko Tanabe², Osamu Kozawa ${ }^{3}$, Hiroki lida²

1 Matsunami General Hospital, Department of Anesthesiology and Critical Care Medicine, Gifu, Japan

2 Gifu University Graduate School of Medicine, Department of Anesthesiology and Pain Medicine, Gifu, Japan

${ }^{3}$ Gifu University Graduate School of Medicine, Department of Pharmacology, Gifu, Japan

\section{Introduction}

\section{Sepsis and Septic DIC}

Disseminated intravascular coagulation (DIC) is most common symptom observed in severe sepsis and septic shock.

Sepsis-induced DIC is characterized by microvasucular thrombosis, reduction of platelets and coagulation inhibitors[1].

\section{Platelet activation}

Collagen is one of most important stimuli for platelet activation.

Activated platelets secrete platelet-derived growth factor (PDGF)-AB and release soluble CD40 ligands (sCD40L). PDGF-AB is a mitogenic mediator and plays a key role in the development of arteriosclerosis[2]. sCD40 ligand is one of the inflammatory mediators in atherosclerosis process[3].

Antithrombin

- Antithrombin (AT) is known as an important physiological anticoagulant.

AT inactivates thrombin and multiple other coagulation factors, thereby AT is used for anticoagulant therapy in sepsis-associated DIC treatment[4].

In our previous studies, we found that pretreatment of AT-III inhibited collagen-stimulated platelet aggregation, secretion of the PDGF-AB and release of SCD40L[5]. However, it is impossible to administrate ATIII before platelets activation as a treatment for septic coagulopathy.

Then, we investigated the effect of AT-III as a treatment for collagenstimulated activated platelets.

\section{Methods}

\section{Subject}

- Twelve healthy volunteers, who have not been taking any antiplatelet medicines, were enrolled in this study.

Platelet preparation and measurement of platelet functions

Blood was centrifuged to separate platelet rich plasma (PRP) and platelet poor plasma (PPP).

PRP was stimulated by $0.5 \mu \mathrm{g} / \mathrm{ml}$ of collagen.

After the PRP stimulation with collagen, AT-III was administrated at $15,30,45,60,90$ or 120 sec later.

Platelet aggregation was recorded by aggregometer.

After recording platelet aggregation, PRP mixture was centrifuged.

Then, the supernatant was subjected to ELISA for PDGF-AB and sCD40L.

Statistical analysis

- Data were analyzed by Student's t-test.

This study was approved by the ethics committee, and written informed consent was obtained.

\section{Results}

Representative data showing the effect of AT-III on the collageninduced platelet aggregation
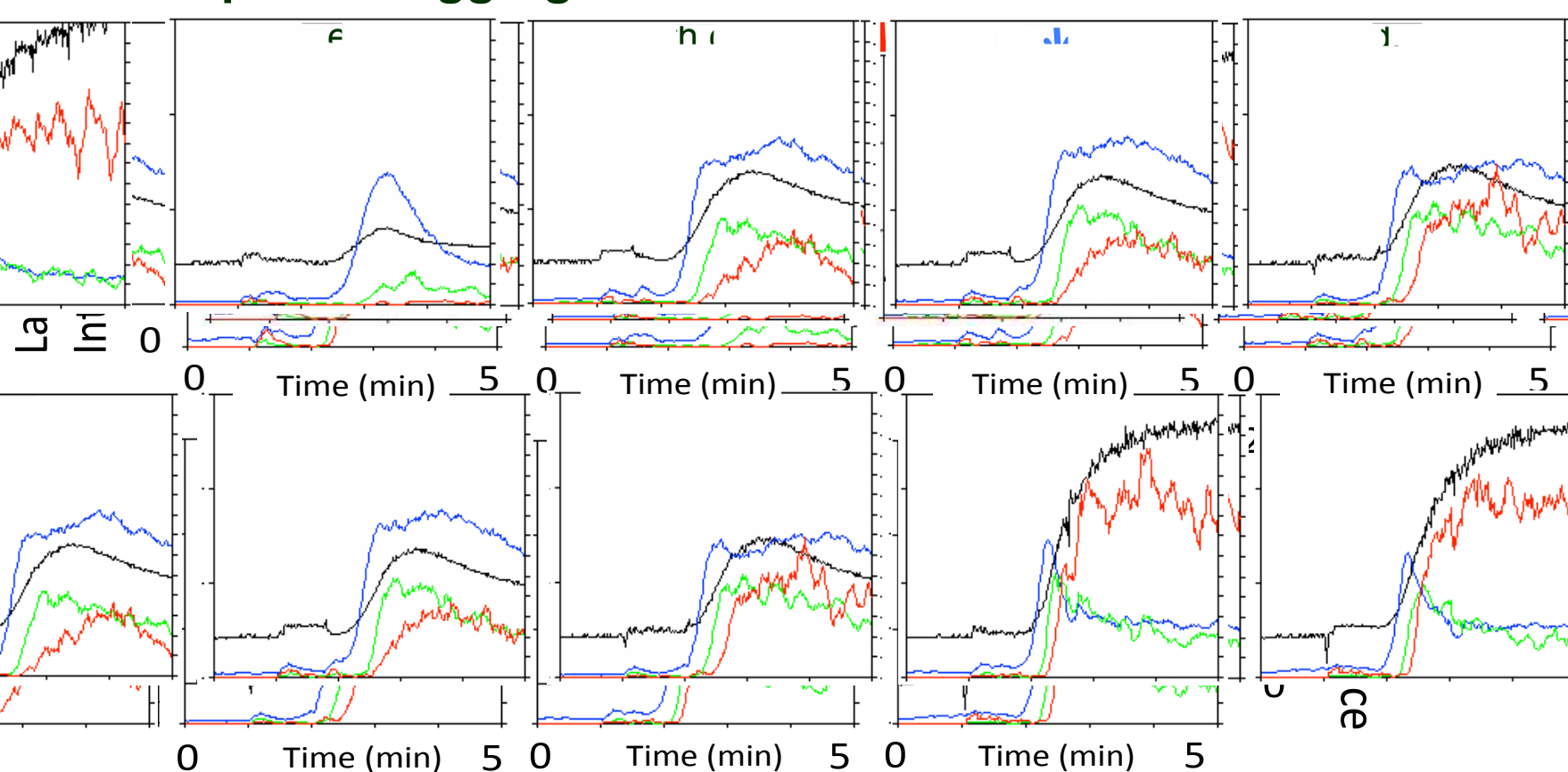

Black line indicates the percentage of transmittance of each sample (the isolated platelets were recorded as $0 \%$, and PPP was recorded as $100 \%)$. Blue line indicates small aggregates $(9-25 \mu \mathrm{m})$ : green line, medium aggregates $(25-50 \mu \mathrm{m})$; red line, large aggregates $(50-70 \mu \mathrm{m})$.

Effect of AT-III on collagen-induced PDGF-AB secretion

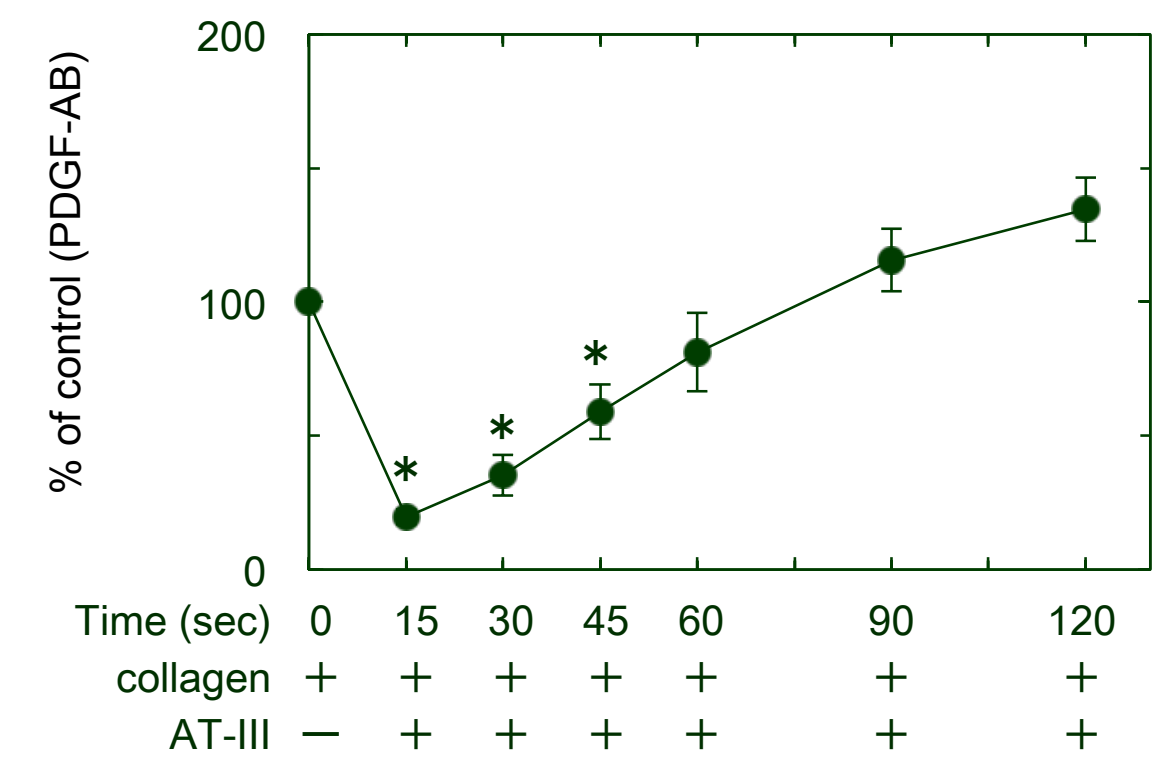

The net increased levels of collagen alone were represented as $100 \%$

The values of the mean \pm SEM were shown.

${ }^{*} p<0.05$, compared with the value of collagen alone.

AT-III administration within $45 \mathrm{sec}$ after collagen stimulation significantly inhibited the secretion of PDGF-AB.

Effect of AT-III on collagen-induced SCD40L release.

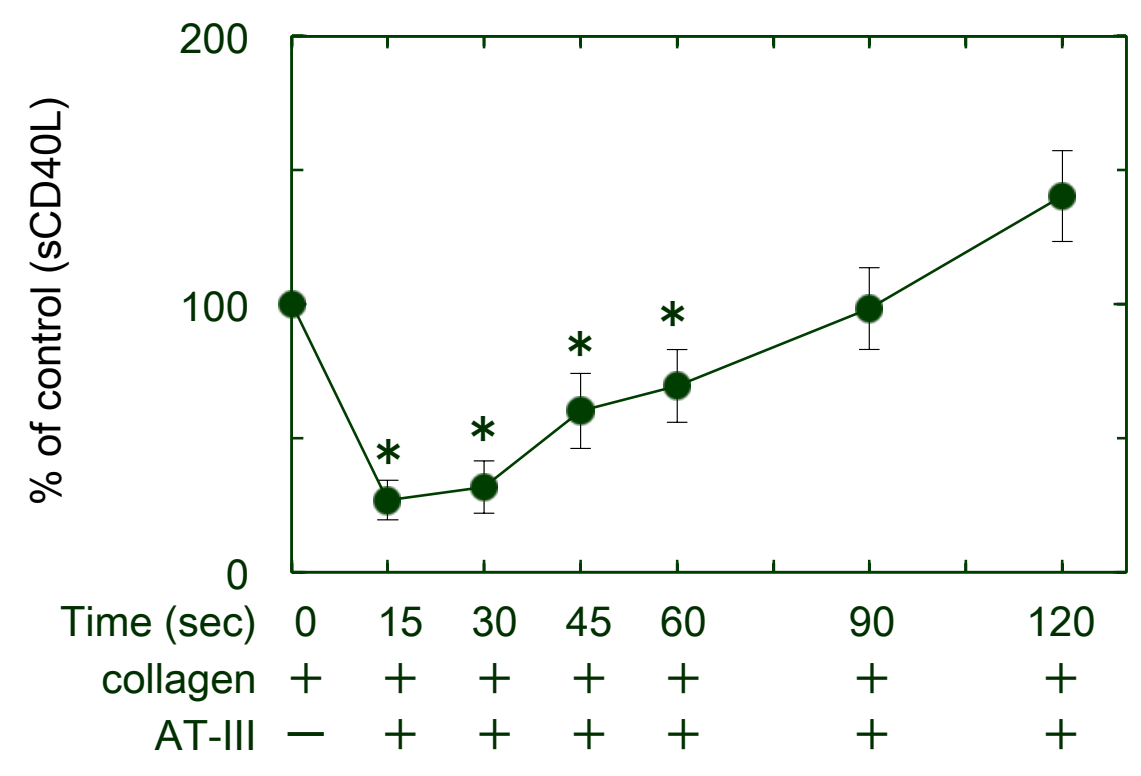

The net increased levels of collagen alone were represented as $100 \%$.

The values of the mean \pm SEM were shown.

${ }^{*} p<0.05$, compared with the value of collagen alone.

AT-III administration within $60 \mathrm{sec}$ after collagen stimulation significantly inhibited the release of SCD40L

\section{Discussion}

AT-III administration after collagen stimulation significantly suppressed platelet aggregation, and also inhibited secretion of the PDGF-AB and release of SCD40L after platelet activation.

$\rightarrow$ It is likely that the effect of AT-III administration suppressed platelets activation after $45-60 \mathrm{sec}$ collagen stimulation.

AT is rapidly depleted in the early phases of sepsis[6].

AT-III treatment was reported to increase survival in sepsis, and AT might be efficacious in sepsis and septic shock[7-10].

$\rightarrow$ Our results can provide a possible explanation of therapeutic effect of AT in sepsis.

We have previously reported suppressive effect of AT-III pretreatment on collagen-stimulated platelet activation [5] In sepsis, all platelets will not be activated at once, but will be activated one after another. Therefore, taken together, AT-III therapy at early period from sepsis onset is considered to be more efficacious.

\section{Conclusion}

In septic coagulopathy, we suggest that administration of AT-III, just after platelet activation, may provide an anti-coagulant and antiinflammatory effect. Starting of AT-III therapy as soon as possible after diagnosis of coagulopathy may be more effective.

\section{References}

N Engl J Med 1999; 341, 586-592 Physiol Rev 1999; 79, 1283-1316 N Engl J Med 2003; 348, 1104-1111 Int J Hematol 2016; 103, 253-61 Int J Mol Med 2010; 26, 387-392
6. Crit Care Med 2000; 28, 34-37

7. JAMA 2001; 286, 1869-1878

8. Crit Care Med 2006; 34, 285-292

9. Thromb Haemost 2006; 95, 850-856

10. Blood Coagul Fibrinolysis 2006; 17, 521-526 